\title{
Article
}

\section{On the Conformal Frames in $f(R)$ Gravity}

\author{
Yuri Shtanov ${ }^{1,2}$ iD
}

check for

updates

Citation: Shtanov, Y. On the Conformal Frames in $f(R)$ Gravity. Universe 2022, 8, 69. https:// doi.org/10.3390/universe8020069

Academic Editor: Panayiotis Stavrinos

Received: 16 December 2021

Accepted: 21 January 2022

Published: 23 January 2022

Publisher's Note: MDPI stays neutral with regard to jurisdictional claims in published maps and institutional affiliations.

Copyright: (C) 2022 by the author. Licensee MDPI, Basel, Switzerland. This article is an open access article distributed under the terms and conditions of the Creative Commons Attribution (CC BY) license (https:// creativecommons.org/licenses/by/ $4.0 /)$.
1 Bogolyubov Institute for Theoretical Physics, Metrologichna St. 14-b, 03143 Kiev, Ukraine; shtanov@bitp.kiev.ua

2 Department of Physics, Taras Shevchenko National University of Kiev, Volodymyrska St. 60, 01033 Kiev, Ukraine

\begin{abstract}
We discuss gravitational physics in the Jordan and Einstein frames of $f(R)$ gravity coupled to the Standard Model. We elucidate the way in which the observed gravitational coupling arises in the Einstein frame for generic $f(R)$. We point out that the effect of "running units" in the Einstein frame is related to the fact that the explicit and implicit quantum parameters of the Standard Model, such as the Higgs vacuum expectation value and the parameter $\Lambda_{\mathrm{QCD}}$, are modified by the conformal transformation of the metric and matter fields and become scalaron-dependent. Considering the scalaron of $f(R)$ gravity describing dark matter, we show that the effect of running units in this case is extremely weak, making two frames practically equivalent.
\end{abstract}

Keyword: modified gravity

\section{Introduction}

Modification of the general relativity theory by considering the Lagrangian in the form of a nonlinear function $f(R)$ of the scalar curvature $R$ is, perhaps, the simplest one and has long been the subject of numerous studies and applications (see [1-3] for reviews). Compared to the general relativity theory, such $f(R)$ gravity contains one extra degree of freedom, which can be used for modelling a wide variety of phenomena, from the inflationary regime in the early universe [4] to dark matter at later epochs [5-11].

The extra degree of freedom is most conveniently identified in the so-called Einstein frame of fields, where it becomes a separate scalar field, called the scalaron, while the remaining gravitational degrees of freedom are described by the general-relativistic action. The existence of different conformal frames has long ago raised the issue of their physical equivalence [12]. After some debates, this question, in principle, appears to have been resolved (see [13]). The conformal frames are physically equivalent and describe the same observable phenomena if one carefully takes into account conformal transformation of all masses. Thus, in the Einstein frame, one then deals with so-called "running units", with all physical masses becoming scalaron-dependent in a universal way.

Although the relation between the conformal frames has been understood in general, it is, perhaps, worth giving it a closer look in the concrete theory of fundamental interactions we are working with. This is the main subject of the present paper, in which we consider the Standard Model minimally coupled to $f(R)$ theory of gravity. In addition, working in the Einstein frame, we are going to estimate potential observable effects of $f(R)$ gravity in a late-time universe in which the oscillating scalaron plays the role of dark matter $[6,11]$.

The Lagrangian of the Standard Model explicitly contains only one dimensionful parameter (in the units $\hbar=c=1$ ), the vacuum expectation value $\eta_{0}$ of the Higgs field. However, according to our current understanding, this Lagrangian is to be regarded as an asymptotic local limit of the renormalisation-group flow. The physics that this theory describes at finite energy scales is characterised also by physical constants arising from the phenomenon known as dimensional transmutation. Most important of these is the parameter $\Lambda_{\mathrm{QCD}}$, which enters the law of renormalisation of the coupling constant of strong interactions. Simplifying things by disregarding other gauge groups, we may take 
that $\Lambda_{\mathrm{QCD}}$ and $\eta_{0}$ determine the masses of all observable particles: hadrons, leptons, and gauge bosons.

We will see that the scalaron in the Einstein frame couples to the Higgs field, in particular, through the scalaron-dependence of the new vacuum expectation value $\widetilde{\eta}(\phi)$. In this sense, the Einstein frame becomes the frame with transformed, or running mass units (in the terminology of [12,13]) for all bare masses of elementary particles, which arise due to the Higgs mechanism. As regards the masses of bound states such as hadrons, they depend in a non-trivial way also on the implicit dimensionful quantum parameters such as $\Lambda_{\mathrm{QCD}}$. This opens up two possibilities of interpreting the theory in the Einstein frame: as a theory with running implicit parameters (which then becomes the frame with running mass units), equivalent to the theory in the original Jordan frame, or as a theory with fixed implicit parameters. Although the two interpretations, in general, differ in their observable predictions, they become equivalent in situations with a completely stabilised or weakly excited scalaron. The last situation arises in a late-time universe in which the excited scalaron plays the role of dark matter [6,11], and we will show that the difference between these interpretations is practically negligible in this case.

\section{Gravitational Constant in the Einstein Frame}

In this section, we review the well-known transition from the Jordan frame to the Einstein frame in the gravity sector. Our attention will be focused on the origin of the gravitational constant (Planck mass) in this frame.

Adopting the metric signature $(-,+,+,+)$, we write the gravitational action in the form:

$$
S_{g}=\int d^{4} x \sqrt{-g} f(R) .
$$

Note that the function $f(R)$ of the scalar curvature has mass dimension four in the unit system $\hbar=c=1$. In general relativity, we have:

$$
f_{\mathrm{GR}}(R)=\frac{M_{\mathrm{P}}^{2}}{3}(R-2 \Lambda),
$$

where $M_{\mathrm{P}}=\sqrt{3 / 16 \pi \mathrm{G}} \approx 3 \times 10^{18} \mathrm{GeV}$ is a conveniently normalised Planck mass (the reason for our choice of this normalisation will become clear below). It is customary to explicitly introduce the factor $M_{\mathrm{P}}^{2} / 3$ in $f(R)$. This can be done without loss of generality, but we will not do this here for better clarity. The point is that any other constant of the same dimension can be factored out here, while our aim is to trace the origin of the physical Planck mass in the Einstein frame.

Proceeding to the Einstein frame, as a first step, one writes action (1) in the form

$$
S_{g}=\int d^{4} x \sqrt{-g}[\Omega R-h(\Omega)]
$$

where $\Omega$ is a new field with mass dimension two, and $h(\Omega)$ is the Legendre transform of $f(R)$. It is defined by the following equations:

$$
\begin{aligned}
& f^{\prime}(R)=\Omega \Rightarrow R=R(\Omega), \\
& h(\Omega)=[\Omega R-f(R)]_{R=R(\Omega)} .
\end{aligned}
$$

The inverse transform allows one to calculate $f(R)$ given $h(\Omega)$; it is obtained by variation of (3) with respect to $\Omega$ :

$$
\begin{aligned}
h^{\prime}(\Omega) & =R \Rightarrow \Omega=\Omega(R), \\
f(R) & =[\Omega R-h(\Omega)]_{\Omega=\Omega(R)} .
\end{aligned}
$$


These transformations may involve subtleties as to which solution is to be chosen in (4) and (6). Solutions of these equations are unique for convex functions, e.g., if $f^{\prime \prime}(R)>0$ everywhere in the domain of validity. In what follows, we will assume $\Omega$ to be positive; therefore, in view of (4), we also require $f^{1}(R)>0$.

Our next step is to transform action (3) so that its linear term in $R$ takes the Einstein form. For this purpose, we perform a conformal transformation of the metric:

$$
g_{\mu \nu}=\frac{M^{2}}{3 \Omega} \widetilde{g}_{\mu \nu}
$$

Here, we explicitly introduced an arbitrary mass parameter $M$, compensating for the dimension of $\Omega$ so that the conformal transformation parameter is dimensionless (leaving the dimension of the metric intact). With this transformation, we have:

$$
\sqrt{-g} \Omega R=\frac{M^{2}}{3} \sqrt{-g} \frac{3 \Omega}{M^{2}} R=\frac{M^{2}}{3} \sqrt{-\widetilde{g}}\left[\widetilde{R}-\frac{3}{2}(\widetilde{\nabla} \ln \Omega)^{2}+3 \widetilde{\square} \ln \Omega\right],
$$

in which all objects related to the new metric $\widetilde{g}_{\mu v}$ are denoted by tildes. The last term is the total derivative and can be dropped. The transformed action (3) then becomes:

$$
S_{g}=\int d^{4} x \sqrt{-\widetilde{g}}\left[\frac{M^{2}}{3} \widetilde{R}-\frac{M^{2}}{2}(\widetilde{\nabla} \ln \Omega)^{2}-W(\Omega)\right]
$$

where

$$
W(\Omega)=\frac{M^{4}}{9} \frac{h(\Omega)}{\Omega^{2}} .
$$

We have obtained an Einstein theory of gravity with a minimally coupled scalar field and with a Planck mass $M$. The theory is stable only if the potential $W(\Omega)$ has a minimum at $\Omega=\Omega_{0}$. In view of system (4)-(7), this condition is equivalent to the existence of $R_{0}$, such that:

$$
R_{0} f^{\prime}\left(R_{0}\right)=2 f\left(R_{0}\right), \quad \frac{1}{f^{\prime \prime}\left(R_{0}\right)}-\frac{R_{0}^{2}}{2 f\left(R_{0}\right)}>0 .
$$

Then, $\Omega_{0}=f^{\prime}\left(R_{0}\right)$. From these relations, it is clear that the values of $\Omega_{0}$ and $R_{0}$ are independent of $M$ introduced in (8). This is also evident from the fact that the parameter $M$ enters only as an overall scaling in potential (11).

We then introduce a scalar field (scalaron) $\phi$ with a canonical kinetic term and with a minimum of potential at $\phi=0$ by setting: ${ }^{2}$

$$
\Omega=\Omega(\phi)=\Omega_{0} e^{\phi / M} .
$$

Action (10), eventually, becomes:

$$
S_{g}=\int d^{4} x \sqrt{-\widetilde{g}}\left[\frac{M^{2}}{3} \widetilde{R}-\frac{1}{2}(\widetilde{\nabla} \phi)^{2}-V(\phi)\right]
$$

where the scalaron potential $V(\phi)$ is calculated by using (5), (11) and (13):

$$
V(\phi) \equiv W(\Omega(\phi))=\frac{M^{4}}{9}\left[\frac{R}{\Omega}-\frac{f(R)}{\Omega^{2}}\right]_{\substack{R=R(\Omega) \\ \Omega=\Omega(\phi)}} .
$$

We note that the mass parameter $M$ has appeared as the Planck mass in action (14), and as an overall scaling in potential (15). ${ }^{3}$ However, this parameter was introduced in (8) quite arbitrarily. This might appear paradoxical, as if $f(R)$ gravity does not predict a specific value for the gravitational constant in the Einstein frame. This paradox is resolved by examining the matter part of the action, which is done in the following section. 


\section{Coupling of Gravity to Matter}

As regards the matter action in the Jordan frame, we take it to be that of the Standard Model minimally coupled to gravity. Proceeding to the Einstein frame via (8) affects this action as well. Note, however, that most of the Standard Model action is classically conformally invariant (with proper conformal transformation of the matter fields), and, therefore, will retain its original form after transformation (8). The only part that breaks conformal invariance is the Higgs sector, with the action:

$$
S_{\mathrm{H}}=-\int d^{4} x \sqrt{-g}\left[g^{\mu \nu}\left(D_{\mu} \Phi\right)^{\dagger} D_{\nu} \Phi+\lambda\left(\Phi^{\dagger} \Phi-\frac{\eta_{0}^{2}}{2}\right)^{2}\right] .
$$

Here, $D_{\mu}$ is the gauge covariant derivative involving the $\mathrm{SU}(2)$ and $\mathrm{U}(1)$ electroweak gauge fields and acting on the Higgs doublet $\Phi$, and $\eta_{0}$ is the symmetry-breaking parameter in the Jordan frame. After the conformal transformation (8), (13) is accompanied by the transformation of the Higgs scalar field:

$$
\Phi=\frac{\sqrt{3 \Omega}}{M} \widetilde{\Phi}
$$

and this action becomes: ${ }^{4}$

$$
\begin{array}{r}
S_{\mathrm{H}}=-\int d^{4} x \sqrt{-\widetilde{g}} \widetilde{g}^{\mu \nu}\left[\left(D_{\mu} \widetilde{\Phi}\right)^{\dagger} D_{\nu} \widetilde{\Phi}+\frac{1}{2 M} \widetilde{\nabla}_{\mu}\left(\widetilde{\Phi}^{\dagger} \widetilde{\Phi}\right) \widetilde{\nabla}{ }_{\nu} \phi+\frac{1}{4 M^{2}} \widetilde{\Phi}^{\dagger} \widetilde{\Phi} \widetilde{\nabla}_{\mu} \phi \widetilde{\nabla} \nu \phi\right] \\
-\lambda \int d^{4} x \sqrt{-\widetilde{g}}\left(\widetilde{\Phi}^{+} \widetilde{\Phi}-\beta e^{-\phi / M} \frac{\eta_{0}^{2}}{2}\right)^{2},
\end{array}
$$

where

$$
\beta=\frac{M^{2}}{3 \Omega_{0}}>0
$$

is a dimensionless constant. We observe the appearance of non-renormalisable interactions of the scalaron $\phi$ with the Higgs field in (18), which, however, are all suppressed by inverse powers of the Planck mass $M$.

From (18), one observes that the Higgs vacuum expectation value in the Einstein frame is:

$$
\widetilde{\eta}(\phi)=\sqrt{\beta} e^{-\phi / 2 M} \eta_{0}
$$

It is this parameter that will determine the bare masses of all fermions and gauge bosons in the model, which are all proportional to it. Now, in the scalaron vacuum $\phi=0$, the ratio of $\widetilde{\eta}_{0}=\widetilde{\eta}(0)=\sqrt{\beta} \eta_{0}$ to the Planck mass $M$ in (14) is:

$$
\frac{\widetilde{\eta}_{0}}{M}=\frac{\eta_{0}}{\sqrt{3 \Omega_{0}}}
$$

This ratio is independent of the chosen scale $M$ in (8), and is uniquely determined by the original Jordan-frame actions (1) and (16). Only this ratio makes sense and is physically measurable. This explains the freedom of choosing $M$ arbitrarily in (8). Assigning the observed value $\widetilde{\eta}_{0} \approx 246 \mathrm{GeV}$, we should then equate $M$ to the Planck mass $M_{\mathrm{P}}=\sqrt{3 / 16 \pi G} \approx 3 \times 10^{18} \mathrm{GeV}$.

The same reasoning applies to the issue of cosmological constant in the Einstein frame. We observe that its purely gravitational contribution $\widetilde{\Lambda}$ in this frame is given by the minimum of the scalaron potential (15):

$$
\widetilde{\Lambda}=\frac{M^{2}}{24} \frac{R_{0}^{2}}{f\left(R_{0}\right)},
$$


where we have used equation (12). We see that the observed ratio $\widetilde{\Lambda} / M^{2}$ is also independent of the chosen scale $M$ in (8). The mass hierarchy problem of modern cosmology can be expressed as:

$$
\frac{\widetilde{\Lambda}}{M^{2}} \ll \frac{\widetilde{\eta}_{0}^{2}}{M^{2}} \ll 1 \Rightarrow \frac{R_{0}^{2}}{f\left(R_{0}\right)} \ll \frac{R_{0} \eta_{0}^{2}}{f\left(R_{0}\right)} \ll 1,
$$

where, we remember, $R_{0}$ is a solution of (12). The last set of inequalities are written in terms of the action in the original Jordan frame.

This analysis would be the whole story for a world described by classical fields. However, the fields in the Standard Model Lagrangian are quantum, and their quantum dynamics are non-trivial.

The Lagrangian of the Standard Model in the Jordan frame contains only one explicit dimensionful parameter, the vacuum expectation value $\eta_{0}$ of the Higgs field. However, according to modern understanding, this Lagrangian is to be regarded as the relevant part of the low-energy (or large-scale) action of some renormalisation-group flow (see, e.g., [18]). The physics that this theory describes at finite energy scales is also characterised by implicit dimensionful parameters arising in what is known as dimensional transmutation. Such is the QCD parameter $\Lambda_{\mathrm{QCD}}$ that enters the law of renormalisation-group flow of the coupling constant of strong interactions and determines the masses of hadrons (see, e.g., $[19,20]$ ). Simplifying the situation by disregarding other gauge interactions, we may take that two dimensionful parameters, $\Lambda_{\mathrm{QCD}}$ and $\eta_{0}$, control the masses of all particles and bound states, including hadrons, in the Jordan frame. With this simplification, we will have, for the $i$ th particle mass:

$$
m_{i}=\Lambda_{\mathrm{QCD}} f_{i}\left(\frac{\eta_{0}}{\Lambda_{\mathrm{QCD}}}\right),
$$

where $f_{i}(x)$ are some dimensionless functions.

This consideration opens up two possibilities of interpreting the theory in the Einstein frame.

\subsection{Einstein Frame with Running Implicit Parameters}

In the matter Lagrangian density, we can proceed to the Einstein frame by the conformal transformation (8) of the metric, scaling the Higgs field and the fermionic fields $\psi$ accordingly:

$$
\mathcal{L}=\mathcal{L}\left(\beta e^{-\phi / M} \widetilde{g}_{\mu v}, \beta^{-1 / 2} e^{\phi / 2 M} \widetilde{\Phi}, \beta^{-3 / 4} e^{3 \phi / 4 M} \widetilde{\psi}\right) \equiv \widetilde{\mathcal{L}}\left(\widetilde{g}_{\mu v}, \widetilde{\Phi}, \widetilde{\psi}, \phi\right)
$$

Here, by virtue of an almost perfect conformal invariance of the action, the last expression differs from the Lagrangian density $\mathcal{L}\left(\widetilde{g}_{\mu \nu}, \widetilde{\Phi}, \widetilde{\psi}\right)$ only in the Higgs part (18). However, the scalaron-dependent scaling of quantum fields, together with (8), lead us to a quantum theory with accordingly scaled, implicit quantum dimensionful parameters. In particular, the QCD parameter in the Einstein frame is locally scaled as:

$$
\widetilde{\Lambda}_{\mathrm{QCD}}(\phi)=\frac{M}{\sqrt{3 \Omega}} \Lambda_{\mathrm{QCD}}=\sqrt{\beta} e^{-\phi / 2 M} \Lambda_{\mathrm{QCD}},
$$

similarly to the scaling (20) of the parameter $\eta_{0}$ in (18). Replacing $\Lambda_{\mathrm{QCD}}$ and $\eta_{0}$ in (24) with their scaled values $\widetilde{\Lambda}_{\mathrm{QCD}}(\phi)$ and $\widetilde{\eta}(\phi)$ in the Einstein frame, we observe that all masses are scaled in the same way:

$$
\widetilde{m}_{i}=\sqrt{\beta} e^{-\phi / 2 M} m_{i} .
$$

Their ratios to the Planck mass $M$ at the scalaron vacuum $\phi=0$ are, again, independent of our choice of $M$ in (8).

Scaling (27) describes the situation of an Einstein frame with running units, an option first discussed in [12] and further elucidated in [13]. Here, the scalaron, in addition to the interaction with the Higgs field through the explicit mass parameter $\widetilde{\eta}(\phi)$ in (18), 
also interacts with matter implicitly via (26) and, therefore, (27). One can arrive at the same picture by considering the matter Lagrangian density in the Einstein frame with transformed metric field only, i.e.,

$$
\mathcal{L}=\mathcal{L}\left(\beta e^{-\phi / M} \widetilde{g}_{\mu v}, \Phi, \psi\right),
$$

and treating $\Lambda_{\mathrm{QCD}}$ and related quantum condensates of the $\psi$ fields as quantities coinciding with their values in the Jordan frame. In this case, one obtains the usual relation between the stress-energy tensors in two frames:

$$
\widetilde{T}_{\mu \nu}=\beta e^{-\phi / M} T_{\mu \nu}
$$

An expression for the mass of a static localised object is:

$$
m_{i}=\int_{\Sigma} T_{\mu \nu} n^{\mu} \xi^{v} d \mu_{\Sigma}
$$

where the integral is taken over a hypersurface $\Sigma$, with $n^{\mu}$ being the vector field of unit normal to this hypersurface; $\xi^{\mu}$ is the timelike killing vector field such that $\xi^{\mu} \xi_{\mu}=-1$ at spatial infinity, and $d \mu_{\Sigma}$ is the volume measure determined by the induced metric on $\Sigma$. Using the conformal transformation laws of these quantities, we again arrive at the transformation law (27) for the mass.

The two (Jordan and Einstein) frames in this interpretation are equivalent; it is a matter of convenience in regards to which frame one chooses to work with, as long as one keeps track of scaling (26) and (27). In particular, all objects (massive as well as massless) move along the geodesics of the Jordan-frame metric $g_{\mu v}=\beta e^{-\phi / M} \widetilde{g}_{\mu v}$, which is the "observable" metric in all respects $[12,13] .^{5}$

\subsection{Einstein Frame with Fixed Implicit Parameters}

Treating the fields in the Lagrangian $\widetilde{\mathcal{L}}\left(\widetilde{g}_{\mu v}, \widetilde{\Phi}, \widetilde{\psi}, \phi\right)$ of (25) as given, and "forgetting" about their Jordan-frame origin, one can specify their quantum theory by a $\phi$-independent implicit quantum parameter $\Lambda_{\mathrm{QCD}}$ in the Einstein frame. This will create a situation quite different from that of Section 3.1, since now, the bare masses of quarks, leptons, and gauge fields will depend on the scalaron, as before, through the Higgs expectation-value parameter (20), while the hadron masses will be given by:

$$
\widetilde{m}_{i}=\Lambda_{\mathrm{QCD}} f_{i}\left(\frac{\widetilde{\eta}(\phi)}{\Lambda_{\mathrm{QCD}}}\right)
$$

depending on the scalaron in a way that is more complicated than (27). We see that, in our framework and strictly speaking, there is no conformal frame with completely fixed units. Particle masses in this frame depend on the scalaron field in a different manner.

For the scalaron in the vacuum, this difference between frames will not be revealed, but it will exist in situations where the scalaron is dynamically excited. One such situation is considered in Section 4.

\subsection{Conformal Anomaly}

The quantum loop corrections to the classical action lead to the effect that the extra degree of freedom present in $f(R)$ gravity couples to matter also due to the conformal anomaly. This is most easily seen in the Einstein frame, in which couplings between the scalaron and gauge fields with strength tensor $F_{\mu \nu}$, for small values of $|\phi| / M$, are proportional to $[6,8]$ :

$$
\alpha_{\mathrm{g}} \frac{\phi}{M} \operatorname{tr} F_{\mu \nu} F^{\mu \nu}
$$

where $\alpha_{\mathrm{g}}=e_{\mathrm{g}}^{2} / 4 \pi$, and $e_{\mathrm{g}}$ is the relevant gauge-coupling parameter. 


\section{Light Scalaron as Dark Matter}

Since the original value of $M$ can be fixed arbitrarily (as was shown Section 3), we fix it in what follows so that $\beta=1$ (this constant is defined in (19)).

The $\phi$-dependence of the particle masses, such as (27) or (31), might be potentially interesting in the case of a classically evolving scalaron. This may lead to important phenomena in the early universe, where the scalaron can be highly excited. For example, if the scalaron plays the role of an inflaton, when proceeding to the Einstein frame, it may be necessary to take into account the dependence (27) of the masses of fundamental particles on the scalaron field during inflation. The same dependence is responsible for particle creation during preheating in such an inflationary theory [14,23].

In this paper, however, we will focus on the situation that arises in a late-time universe in which the oscillating scalaron plays the role of dark matter [6,11]. In this case, the scalaron oscillations might lead to potentially observable effects. Such effects are determined by the small ratio: 6

$$
\frac{|\phi|}{M} \simeq \frac{\sqrt{2 \rho_{\mathrm{s}}}}{M^{2}} \frac{M}{m} \simeq 10^{-33}\left(\frac{\rho_{\mathrm{s}}}{\bar{\rho}_{\mathrm{s}}}\right)^{1 / 2}(1+z)^{3 / 2} \frac{\mathrm{eV}}{m},
$$

where $z$ is the cosmological redshift, $\rho_{\mathrm{s}}$ is the local scalaron energy density, $\bar{\rho}_{\mathrm{s}}$ is its spatial average in the universe, and $m$ is the scalaron mass. The effects are very small because the scalaron mass in the interpretation of the Einstein frame with running units is bounded from below by non-observation [25,26] of the additional Yukawa forces [27] between nonrelativistic masses (see also $[6,28]$ ):

$$
m \geq 2.7 \times 10^{-3} \mathrm{eV} \quad \text { at } 95 \% \text { C.L. }
$$

As regards the Einstein frame with constant implicit parameter $\Lambda_{\mathrm{QCD}}$, the $\phi$-dependence in (31) will have additional smallness because the contribution of the bare quark masses to the masses of hadrons constitute only a tiny fraction of the total mass [19].

Let us examine this in more detail for the late-time universe. The effect is most prominent in the interpretation of the Einstein frame with running units, where the observable metric will be that of the Jordan frame. Then, in the Einstein frame, all masses scale with the scalaron as (27). This produces second-order effects in the small gravitational potential because of the smallness of the ratio (33). However, in the observable metric, this gives an effect of the first order because of the scaling $g_{\mu \nu}=e^{-\phi / M} \widetilde{g}_{\mu \nu}$. This will not affect null geodesics apart from additional redshift, but will produce an effective Newtonian potential,

$$
\varphi_{\mathrm{eff}}=\varphi-\frac{\phi}{2 M}
$$

for non-relativistic matter. Here, $\varphi$ is the Newtonian potential in the Einstein-frame metric $\widetilde{g}_{\mu \nu}$, which is determined by the distribution of the usual matter and dark matter in the form of scalaron. In the Newtonian approximation to gravity, which is of relevance here, it is described by the Poisson equation $\nabla^{2} \varphi=3 \rho / 4 M^{2}$, where $\rho$ is the total energy density of matter, including the scalaron field.

The scalaron field $\phi$ of mass $m$ is oscillating in time with the following period:

$$
t=\frac{2 \pi}{m} \approx 4.1 \times 10^{-15}\left(\frac{\mathrm{eV}}{m}\right) \mathrm{s}
$$

Therefore, the last term in the effective Newtonian potential (35) is rapidly oscillating in time with space-dependent amplitude. Since one is interested in the motion of astrophysical bodies on time scales much longer than (36), one should perform time averaging of their dynamics in such a rapidly oscillating potential. The solution to this classical problem is 
well known (see $\$ 30$ in [29]). The average long-term effective potential acting on test bodies is given by:

$$
\bar{\varphi}_{\mathrm{eff}}=\varphi+\frac{\overline{(\nabla \phi)^{2}}}{8 m^{2} M^{2}}=\varphi+\frac{\left(\nabla \phi_{0}\right)^{2}}{16 m^{2} M^{2}}
$$

where overline denotes averaging over a period of oscillations of $\phi$, and $\phi_{0}$ is the spacedependent amplitude of its oscillations. Since the scalaron energy density $\rho_{\mathrm{s}}=\frac{1}{2} m^{2} \phi_{0}^{2}$, we have:

$$
\bar{\varphi}_{\text {eff }}=\varphi+\frac{\left(\nabla \rho_{\mathrm{s}}\right)^{2}}{32 m^{4} M^{2} \rho_{\mathrm{s}}}=\varphi+\frac{\left(\nabla \nabla^{2} \varphi_{\mathrm{s}}\right)^{2}}{24 m^{4} \nabla^{2} \varphi_{\mathrm{s}}},
$$

where we have used the Poisson equation $\rho_{\mathrm{S}}=\frac{4}{3} M^{2} \nabla^{2} \varphi_{\mathrm{s}}$ for the scalaron contribution $\varphi_{\mathrm{s}}$ to the total gravitational potential $\varphi$.

The scalaron energy density, and the gravitational potential $\varphi_{s}$, hence, varies on the spatial scale of the de Broglie wavelength (see [30] for a review on such wave dark matter):

$$
\lambda_{\mathrm{dB}} \simeq \frac{2 \pi}{m v}=124\left(\frac{10^{-3} \mathrm{eV}}{m}\right)\left(\frac{10^{-3}}{v}\right) \mathrm{cm},
$$

where $v$ is the velocity dispersion in a virialised dark-matter halo (in units of the speed of light). For the last term in (38), this gives an estimate:

$$
\frac{\left(\nabla \nabla^{2} \varphi_{\mathrm{s}}\right)^{2}}{24 m^{4} \nabla^{2} \varphi_{\mathrm{s}}} \sim \frac{\left|\varphi_{\mathrm{s}}\right|}{24 m^{4} \lambda_{\mathrm{dB}}^{4}} \sim 10^{-4} v^{4}\left|\varphi_{\mathrm{s}}\right| .
$$

Here, we have replaced all spatial gradients by the characteristic inverse length $\lambda_{\mathrm{dB}}^{-1}$. For typical velocity dispersions $v \sim 10^{-2}-10^{-3}$, this is many orders of magnitude smaller than $\left|\varphi_{\mathrm{s}}\right|$. The de Broglie wavelength scale (39) and time scale $t_{\mathrm{dB}}=\lambda_{\mathrm{dB}} / v$ themselves are rather small for masses $m \gtrsim 10^{-3} \mathrm{eV}$ allowable in this theory. The direct effects of the scalaron oscillations are, thus, quite negligible, and the Jordan and Einstein conformal frames are practically indistinguishable in this case.

Couplings (32) due to the conformal anomaly lead to the scalaron decay into photons, with lifetime $\tau \sim M^{2} / \alpha^{2} m^{3} \sim 10^{36}(\mathrm{eV} / m)^{3} \mathrm{yr}$, exceeding the age of the universe $\left(1.4 \times 10^{10} \mathrm{yr}\right)$ for $m \ll 10^{8} \mathrm{eV}$. (Here, $\alpha$ is the fine-structure constant.) For the scalaron masses of order $m \sim 10^{-3} \mathrm{eV}$, as in the scenario of [11], such a light scalaron dark matter appears to be quite "sterile" and hard to detect by means other than gravitationally. The smallness of the specific gravitational manifestations in the scenario under consideration would make it very difficult to establish that we are dealing with $f(R)$ gravity. Perhaps, this could be done only by detecting a specific Yukawa contribution to gravitational forces at submillimetre spatial scales [25-28].

\section{Discussion}

A generic gravity theory with action (1) has a stable point if there exists a solution to (12). In this case, it is conformally transformed by (8) to a general-relativistic theory (14) with a minimally coupled scalar field with potential (15) that has minimum at $\phi=0$. The gravitational coupling at this stage is introduced arbitrarily in (8). However, its physical value in the Einstein frame is uniquely determined by the function $f(R)$, together with the matter action in the Jordan frame. This was demonstrated in Section 3, where we considered the action of the Standard Model minimally coupled to gravity in the Jordan frame.

We reviewed and further elucidated two possible interpretations of this model in the Einstein frame, namely, as the frame with either running or constant implicit quantum parameters. The Standard Model contains at least one such implicit parameter $\Lambda_{\mathrm{QCD}}$, which transforms under conformal transformation of the metric. The difference between these 
two interpretations, however, is practically insignificant in situations where the scalaron field is weakly excited. We verified this for the case of cosmology in which a light scalaron plays the role of dark matter. In such a scenario, the fact that we are dealing with $f(R)$ gravity could be observationally verified, perhaps, only by detecting a specific Yukawa contribution to gravitational forces at submillimetre spatial scales [25-28].

Funding: This research was funded by the National Academy of Sciences of Ukraine project number $0121 \mathrm{U} 109612$.

Institutional Review Board Statement: Not applicable.

Informed Consent Statement: Not applicable.

Data Availability Statement: No new data were created or analysed in this study. Data sharing is not applicable to this article.

Acknowledgments: The author is grateful to Maria Khelashvili and Anton Rudakovskyi for discussion.

Conflicts of Interest: The author declares no conflict of interest. The funders had no role in the design of the study; in the collection, analyses, or interpretation of data; in the writing of the manuscript, or in the decision to publish the results.

\title{
Abbreviations
}

The following abbreviations are used in this manuscript:

\author{
C.L. Confidence Level \\ GR General Relativity \\ QCD Quantum Chromodynamics
}

\section{Notes}

This requirement ensures that the effective gravitational coupling is positive in the Jordan frame, see [1-3] for reviews.

If $W(\Omega)$ does not have a minimum, we still can write (13) by choosing $\Omega_{0}$ arbitrarily. In this case, however, the theory will not have a stable point.

3 The appearance of a simple expression $\phi / M$ (without numerical factor) in the exponent (13) and in all subsequent exponents of this type is the reason why we have chosen the particular normalisation constant in (8) and in (2).

4 A similar result of the conformal transformation of fields was under consideration, e.g., in [14-16]. In passing, we note that conformal transformation, in our theory, where the Higgs scalar field is minimally coupled to gravity, looks much simpler compared to the case of its non-minimal coupling, as is the case, e.g., in the model of Higgs inflation [17].

5 In the context of scalar-tensor theories, such an equivalence between frames on the tree level was recently demonstrated in [16]. On the one-loop level, on-shell equivalence between conformal frames was demonstrated previously in [21,22].

6 For the solar neighbourhood, the dark-matter density is $\rho_{\mathrm{dm}} \simeq 10^{-2} M_{\odot} / \mathrm{pc}^{3}$ [24], which gives $\left(\rho_{\mathrm{s}} / \bar{\rho}_{\mathrm{s}}\right)^{1 / 2} \simeq 500$.

\section{References}

1. Sotiriou, T.P.; Faraoni, V. $f(R)$ theories of gravity. Rev. Mod. Phys. 2010, 82, 451-497. [CrossRef]

2. De Felice, A.; Tsujikawa, S. $f(R)$ Theories. Living Rev. Rel. 2010, 13, 3. [CrossRef]

3. Nojiri, S.; Odintsov, S.D.; Oikonomou, V.K. Modified Gravity Theories on a Nutshell: Inflation, Bounce and Late-time Evolution. Phys. Rept. 2017, 692, 1-104. [CrossRef]

4. Starobinsky, A.A. A new type of isotropic cosmological models without singularity. Phys. Lett. B 1980, 91, 99-102. [CrossRef]

5. Nojiri, S.; Odintsov, S.D. Dark energy, inflation and dark matter from modified F(R) gravity. TSPU Bull. 2011, N8, 7-19. arXiv:0807.0685.

6. Cembranos, J.A.R. Dark Matter from $R^{2}$ Gravity. Phys. Rev. Lett. 2009, 102, 141301. [CrossRef] [PubMed]

7. Corda, C.; Mosquera Cuesta, H.J.; Lorduy Gómez, R. High-energy scalarons in $R^{2}$ gravity as a model for Dark Matter in galaxies. Astropart. Phys. 2012, 35, 362-370. [CrossRef]

8. Katsuragawa, T.; Matsuzaki, S. Dark matter in modified gravity? Phys. Rev. D 2017, 95, 044040. [CrossRef]

9. Yadav, B.K.; Verma, M.M. Dark matter as scalaron in $f(R)$ gravity models. J. Cosmol. Astropart. Phys. 2019, 10, 052. [CrossRef]

10. Parbin, N.; Goswami, U.D. Scalarons mimicking dark matter in the Hu-Sawicki model of $f(R)$ gravity. Mod. Phys. Lett. A 2021, 36, 2150265. [CrossRef]

11. Shtanov, Y. Light scalaron as dark matter. Phys. Lett. B 2021, 820, 136469. [CrossRef] 
12. Dicke, R.H. Mach's principle and invariance under transformation of units. Phys. Rev. 1962, 125, 2163-2167. [CrossRef]

13. Faraoni, V.; Nadeau, S. The (pseudo)issue of the conformal frame revisited. Phys. Rev. D 2007, 75, 023501. [CrossRef]

14. Rudenok, I.; Shtanov, Y.; Vilchinskii, S. Post-inflationary preheating with weak coupling. Phys. Lett. B 2014, 733, 193-197. [CrossRef]

15. Burrage, C.; Copeland, E.J.; Millington, P.; Spannowsky, M. Fifth forces, Higgs portals and broken scale invariance. J. Cosmol. Astropart. Phys. 2018, 11, 036. [CrossRef]

16. Copeland, E.J.; Millington, P.; Muñoz, S.S. Fifth forces and broken scale symmetries in the Jordan frame. arXiv 2021, arXiv:2111.06357.

17. Bezrukov, F.L.; Shaposhnikov, M. The Standard Model Higgs boson as the inflaton. Phys. Lett. B 2008, 659, 703-706. [CrossRef]

18. Costello, K. Renormalization and Effective Field Theory; Mathematical Surveys and Monographs 170; American Mathematical Society: Providence, RI, USA, 2011; 251p.

19. Chivukula, R.S. The Origin of mass in QCD. eConf 2004, C040802, L010.

20. Deur, A.; Brodsky, S.J.; de Teramond, G.F. Connecting the hadron mass scale to the fundamental mass scale of quantum chromodynamics. Phys. Lett. B 2015, 750, 528-532. [CrossRef]

21. Kamenshchik, A.Y.; Steinwachs, C.F. Question of quantum equivalence between Jordan frame and Einstein frame. Phys. Rev. D 2015, 91, 084033. [CrossRef]

22. Ruf, M.S.; Steinwachs, C.F. Quantum equivalence of $f(R)$ gravity and scalar-tensor theories. Phys. Rev. D 2018, 97, 044050. [CrossRef]

23. Gorbunov, D.S.; Panin, A.G. Scalaron the mighty: Producing dark matter and baryon asymmetry at reheating. Phys. Lett. B 2011, 700, 157-162. [CrossRef]

24. Read, J.I. The local dark matter density. J. Phys. G 2014, 41, 063101. [CrossRef]

25. Kapner, D.J.; Cook, T.S.; Adelberger, E.G.; Gundlach, J.H.; Heckel, B.R.; Hoyle, C.D.; Swanson, H.E. Tests of the gravitational inverse-square law below the dark-energy length scale. Phys. Rev. Lett. 2007, 98, 021101. [CrossRef]

26. Adelberger, E.G.; Heckel, B.R.; Hoedl, S.A.; Hoyle, C.D.; Kapner, D.J.; Upadhye, A. Particle-physics implications of a recent test of the gravitational inverse-square law. Phys. Rev. Lett. 2007, 98, 131104. [CrossRef]

27. Stelle, K.S. Classical gravity with higher derivatives. Gen. Rel. Grav. 1978, 9, 353-371. [CrossRef]

28. Perivolaropoulos, L.; Kazantzidis, L. Hints of modified gravity in cosmos and in the lab? Int. J. Mod. Phys. D 2019, 28, 1942001. [CrossRef]

29. Landau, L.D.; Lifshitz, E.M. Mechanics. Course of Theoretical Physics; Butterworth-Heinemann: Oxford, UK, 1976 ; Volume 1.

30. Hui, L. Wave Dark Matter. Annu. Rev. Astron. Astrophys. 2021, 59, 247-289. [CrossRef] 